\title{
Finite element simulation of laser transmission welding of dissimilar materials between polyvinylidene fluoride and titanium
}

\author{
Bappa Acherjee $^{1 *}$, Arunanshu S. Kuar ${ }^{1}$, Souren Mitra ${ }^{1}$, Dipten Misra ${ }^{2}$ \\ ${ }^{1 *}$ Department of Production Engineering, Jadavpur University, Kolkata - 700 032, INDIA \\ ${ }^{2}$ School of Laser Science \& Engineering, Jadavpur University, Kolkata - 700 032, INDIA \\ *Corresponding author: email: a.bappa@yahoo.com, bappa.rana@gmail.com
}

\begin{abstract}
Now-a-days, metal to plastic micro-welding is of great interest in the field of biomedical and electronics applications. Laser transmission welding (LTW) has emerged as the most suitable technique for such applications. In this paper, a three-dimensional finite element (FE) thermal model is developed to simulate the laser transmission welding process for joining polyvinylidene fluoride to titanium using a distributed moving heat flux. The objectives of this study are to predict the transient temperature field as well as the weld dimensions. All the major physical phenomena associated with the LTW process, such as, heat radiation, thermal conduction and convection heat losses are taken into account in the model development. The simulation algorithm is programmed as a macro routine within the ANSYS $^{\circledR}$ finite element code. The developed model derives its main advantage from its applicability in parametric studies of a wide range of laser transmission metal to plastic welding problems of different geometrical, material and joint type, requiring only the basic thermo-physical material properties, the geometric details and the laser process parameters as input.
\end{abstract}

Keywords: Laser transmission welding, Temperature field, Weld dimension, Finite element analysis, Thermal modeling

\section{Introduction}

Laser transmission welding is a novel and promising technology for many industries, such as, automotive, electronic, and medical industry. The electronics and medical devices industries employ micro-joining of dissimilar materials for the majority of their applications. Joining of dissimilar materials requires the application of an appropriate joining technology. In joining biomedical products, the joining process should not make use of any third material, which is not biocompatible. The laser transmission welding process meets this condition. Being a non-contact process, laser transmission welding does not lead to contamination at the functional areas of the bio-medical products (Sultana et al., 2009). In this laser welding process a laser beam is passed through one transparent material, directly heating only the second material, precisely at the mating surface. With this technique, melt is only created where it is needed, in the joining area of both partners, reducing the stress into the joining materials to a minimum. Furthermore, this joining process is flexible, has shorter processing time, can provide consistent quality and is repeatable.

The efficiency of laser transmission welding strongly depends on the optical properties of the joining materials and the process parameters. The process parameters control the temperature field inside the weld seam during welding, which plays very significant role in determining the shape and strength of the weld seam (Acherjee et al., 2009). It is difficult to measure the temperature field of welding. It always needs many prior tests to set down suitable laser welding process for some materials requiring a great deal of manpower, material and financial resources. With the progress of improved technology in the software and hardware, the virtual technology of manufacture gives rise to the upsurge, including the numerical simulation of heat-process in welding.

In this decade, the numerical simulation of laser transmission welding process has gained a great interest among the researchers for studying various aspects of this novel plastic joining technique. Kennish et al. (2002) developed an analytical heat transfer model of LTW to predict the process capabilities and weld characteristics. Their model gives an approximate solution for peak 
temperature at the weld interface as a function of depth. Becker et al. (2002) studied the heating phase of laser transmission welding of polypropylene using finite element method. Ilie et al. (2005) presented a study on effects of laser beam scattering phenomena in a semi-transparent polymer induced by their compositions in the LTW process. Mayboudi et al. (2005) developed a two-dimensional heat transfer model of LTW of unreinforced nylon 6 in a T-like joint geometry. Van de Ven and Erdman (2007) set a two-dimensional heat transfer model to study the LTW process of polyvinyl chloride. A three-dimensional transient thermal model of LTW solved with FEM is presented by Mayboudi et al. (2007) for PA6 with lap joint geometry. They considered a stationary rectangular laser beam with uniform power density. In their following work, Mayboudi et al. (2007) developed a three dimensional thermal model for LTW considering a moving laser beam. They modelled a 3-D non-Gaussian beam profile including the effect of laser beam scattering by the top transparent part. This model is found to be capable of predicting temperature distribution along the weld line as well as molten depth. Coelho et al. (2008) studied the beam spot influence in high speed laser lap welding of thermoplastic films. Recently, Taha et al. (2009) developed a novel mathematical model for LTW considering the effect of clamp pressure on the weld geometry and thermal profile. They considered a contact resistance between the two sections in lap joint configuration due to the possible air gap at interface. They quantified the contact resistance by an average heat transfer coefficient, which is estimated by matching the weld pool dimensions predicted by the model with that obtained experimentally as a function of applied pressure.

The present work contributes to the development of a three-dimensional heat transfer model, for laser assisted metal to plastic welding process, that can predict the transient temperature field as well as the weld dimensions. The finite element model is parametrically built using a scripting language, APDL (ANSYS ${ }^{\circledR}$ Parametric Design Language) provided by the commercial code ANSYS 10. The thermal model encompasses the whole laser transmission welding process including the heating and cooling phases with a moving heat flux.

\section{Materials and Process}

The ideal material combination to be joined using laser transmission welding process includes one absorbent and one transparent part. The laser energy penetrates the transparent part and is absorbed by the absorbing part. The laser energy in the near-infrared spectrum that is absorbed in the absorbing part causes excitation of valence and conduction band electrons. This electron-phonon interaction at the absorbing surface causes localized heating of the material (Datta Majumdar and Manna, 2003). This heat is conducted within and between both parts and transferred to the surroundings through convection and radiation. In addition, appropriate temperature development at the laser irradiation zone results in chemical bonds between the joining materials.

In this study, laser transmission welding of polyvinylidene fluoride (PVDF) foil to titanium (Ti) foil by means of continuous wave radiation from a diode laser at $\lambda=804.9 \mathrm{~nm}$ is considered. PVDF is a flexible polymer, which has a high piezoelectric coefficient, and low acoustic and mechanical impedance. These properties make the PVDF a very desirable material for microelectro-mechanical systems (MEMS) applications. PVDF is also a biocompatible material. It is a biomaterial established for soft tissue applications and as a suture material. It is chemical and weather resistant, durable and has very low water absorption. PVDF can be considered as a potential material for application in biomedical devices. Even though PVDF possesses many desirable properties, it is known that the adhesion and bonding of metals to this polymer is difficult, because of the inert surface of the polymer. Earlier studies show that reactive metals (such as $\mathrm{Cr}$, Ti, Ta and $\mathrm{Al}$ ) react extensively with fluoropolymer surfaces, and form sharp and thermally stable interfaces (Sultana et al., 2008).

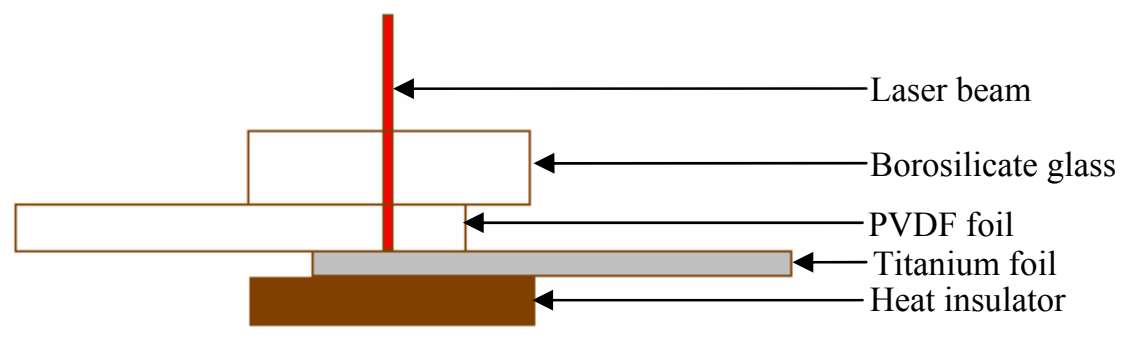

Figure 1. Schematic diagram of laser transmission welding process

Figure 1 shows a schematic representation of the laser transmission welding process considered for the present numerical simulation. The PVDF film of $0.13 \mathrm{~mm}$ thickness is laser welded to a $0.05 \mathrm{~mm}$ Ti foil by using a laser power of $4 \mathrm{~W}$ and a welding speed of $800 \mathrm{~mm} / \mathrm{min}$. The laser beam is focused on the Ti surface to a spot with diameter of $400 \mu \mathrm{m}$. We assume an optimum weld pressure is applied on the mating parts, which ensures a perfect contact at the weld interface. The geometric model is created by considering that both the materials are in perfect contact at the interface.

The transient temperature field generated during the laser transmission welding is determined based on the mechanism of heat conduction. The following assumptions are made for the calculation of temperature: 
1) The laser intensity distribution follows the Gaussian mode.

2) Heat conduction within the specimens and free convection between the surfaces of the specimen and the surrounding air are considered.

3) The thermal radiation from the top surface of the Ti foil is considered.

4) The heating phenomena due to the phase changes are neglected.

\section{The Simulation of Temperature Field in Laser Welding}

\subsection{Governing equation}

The following three-dimensional heat conduction equation is considered to model the heat transfer in laser transmission welding process that defines the temperature distribution within the body, based on the energy conservation law, which balances the rate of the internally generated heat within the body, body's capacity to store this heat, and the rate of thermal conduction to the boundaries (Incropera and DeWitt, 1996):

$$
\frac{\partial}{\partial x}\left(k_{x} \frac{\partial T}{\partial x}\right)+\frac{\partial}{\partial y}\left(k_{y} \frac{\partial T}{\partial y}\right)+\frac{\partial}{\partial z}\left(k_{z} \frac{\partial T}{\partial z}\right)+q(x, y, z, t)=\rho C \frac{\partial T}{\partial t}
$$

where, $k_{x}, k_{y}, k_{z}$ are the thermal conductivity in the $x, y$ and $z$ directions $(\mathrm{W} / \mathrm{m}-\mathrm{K}), C$ is the specific heat capacity $(\mathrm{J} / \mathrm{kg}-\mathrm{K}), \rho$ is the density $\left(\mathrm{kg} / \mathrm{m}^{3}\right), t$ is the time (s) and $q(x, y, z, t)$ is internal heat generation rate per unit volume $\left(\mathrm{W} / \mathrm{m}^{3}\right)$.

\subsection{The material property}

The accuracy of numerical results depends directly on a good evaluation of thermo-physical and optical characteristics. The material properties required for the heat flow analysis are thermal conductivity, specific heat and density for all of the materials all of which are assumed to be constant and the thermal conductivity is considered as isotropic. The material properties are obtained from www.matweb.com and are given in Table 1.

Table 1. Thermal properties of the materials used in the FE model

\begin{tabular}{|l|c|c|c|c|}
\hline Material & $\begin{array}{c}\text { Density, } \rho \\
\left(\mathrm{kg} / \mathrm{m}^{3}\right)\end{array}$ & $\begin{array}{c}\text { Thermal conductivity, } k \\
(\mathrm{~W} / \mathrm{m}-\mathrm{K})\end{array}$ & $\begin{array}{c}\text { Specific heat, } C \\
(\mathrm{~J} / \mathrm{kg}-\mathrm{K})\end{array}$ & $\begin{array}{c}\text { Melting point, } t_{m} \\
(\mathrm{~K})\end{array}$ \\
\hline PVDF & 1790 & 0.13 & 1214 & 443 \\
\hline Titanium & 4440 & 17 & 530 & 1998 \\
\hline
\end{tabular}

\subsection{Creating model}

Due to the presence of symmetry in the geometry and laser beam profile, the geometry is cut in the laser beam symmetry plane (Y-Z plane) as shown in the figure 2. Employing symmetry to reduce model geometry reduces simulation time. This idealization of the model is adequate to represent the problem to achieve study goals.

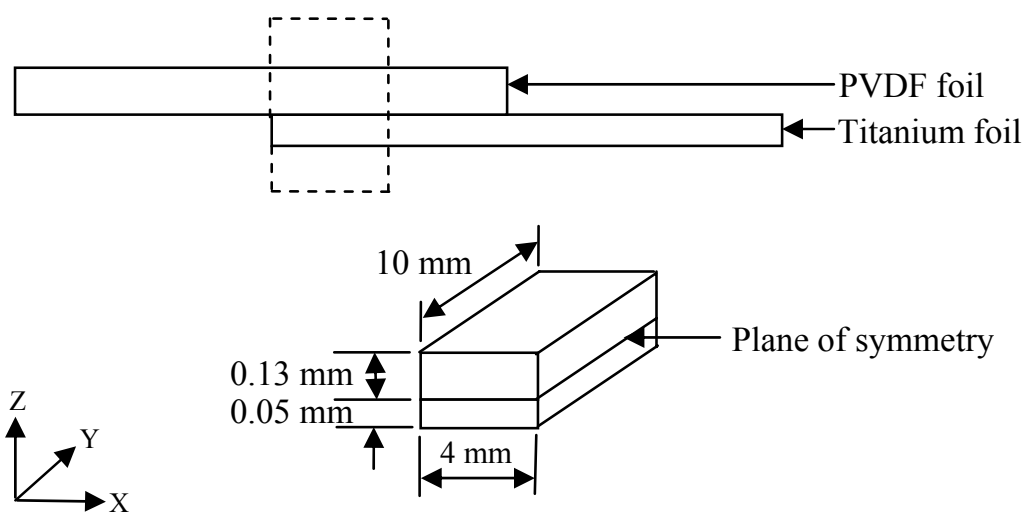

Figure 2. Schematic of the sample and one half of the model at the bond region with symmetric boundary conditions

In the present thermal analysis, the model for PVDF and Ti foils is meshed using thermal brick elements, called Solid 70 (ANSYS ${ }^{\mathbb{B}} 10$ Manual), which has eight nodes with a single degree of freedom, temperature, at each node. The element has also prism and tetrahedral options. In addition to that, the top surface of Ti foil is meshed with a set of radiation elements, Surf 152 , overlaid onto the 3-D thermal elements. A non-uniform mesh pattern is generated: fine meshes are created in the region near the laser path due to the high heat flux involved in that portion; less fine meshes in the neighboring zone of the fine meshes; and coarse meshes in the outer portion of the model, as shown in figure 3 . With this mesh pattern, accurate result of the simulation as 
well as reduction of simulation time can be achieved. A non-uniform mesh pattern and the tapered element for the transitional meshes cannot be directly produced from an ANSYS $^{\mathbb{B}}$ command, so that, the corresponding nodes to the mesh pattern are generated first, then the elements are directly created using these nodes. The quality of the mesh is confirmed by a series of tests increasing the mesh density until the temperatures calculated are consistent.

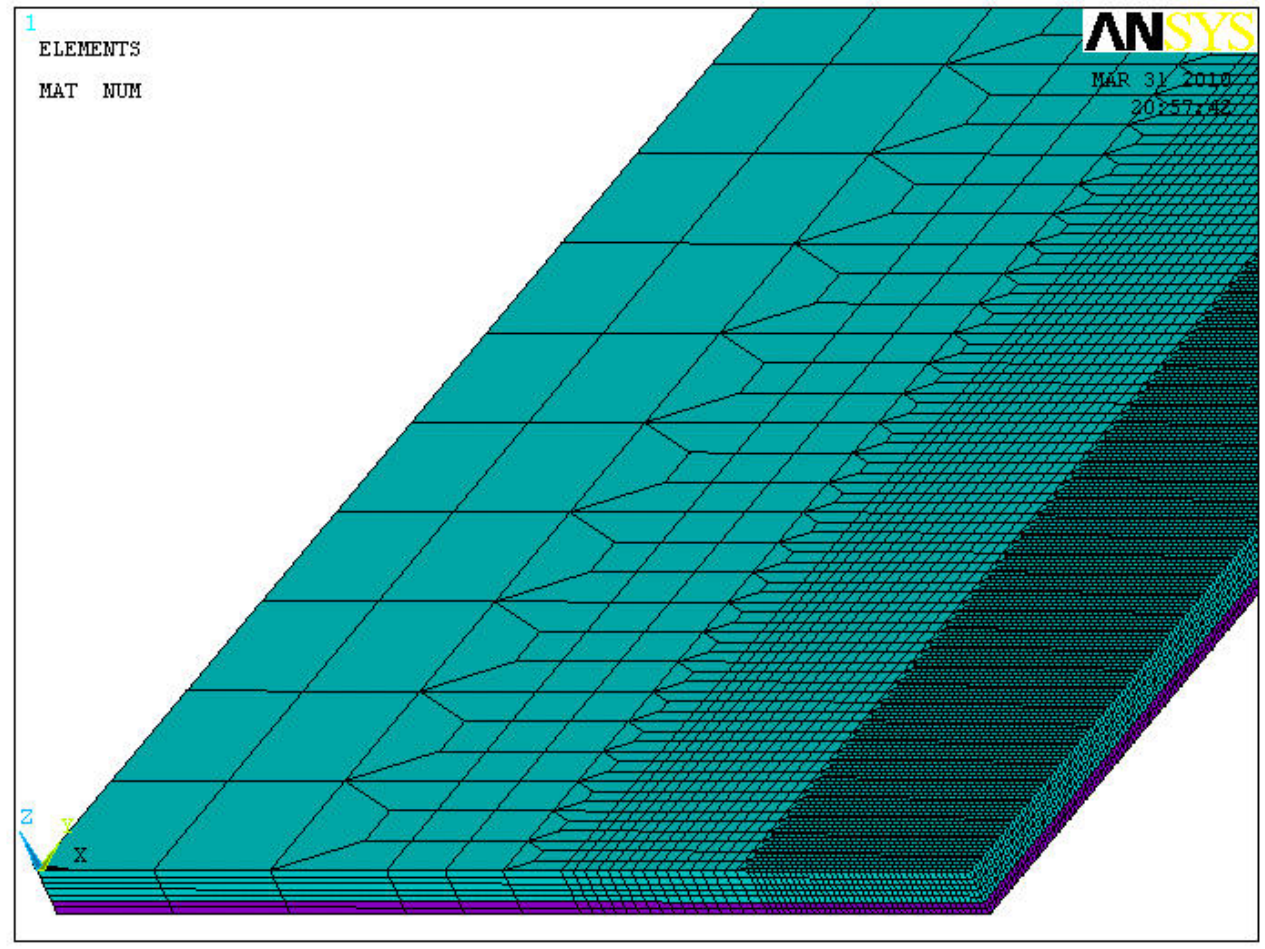

Figure 3. Finite element mesh of the simulation domain

\subsection{Heat flux}

The laser energy directed to the overlapping PVDF-Ti foils, penetrates the top transparent PVDF part and is absorbed by the absorbing Ti part within a thickness of less than a micron and transferred to heat. Hence for this case, it is quite reasonable to assume that the absorbed laser energy is converted into heat at the surface itself, and the laser beam may be considered to be equivalent to a surface heat flux. The heat flux for the present study is modeled with a Gauss-shaped heat flux whose intensity varies with the beam radius. For the case of Gaussian power distribution, the equation for the incident heat flux $q\left(\mathrm{~W} / \mathrm{m}^{2}\right) \mathrm{can}$ be expressed as:

$$
q=\frac{2 A P}{\pi r_{0}^{2}} \exp \left(-\frac{2 r^{2}}{r_{0}^{2}}\right)
$$

where, $A$ is the fraction of laser energy absorbed in the bottom (Ti) material, $P$ is the laser beam power, $r_{0}$ is the laser beam radius, $r=\sqrt{ }\left(x_{s}{ }^{2}+y_{s}{ }^{2}\right)$ is the radial distance of any point from beam center on the surface of the material, where $x_{s}$ and $y_{s}$ are the Cartesian coordinates of that point. The parameter $A$ is calculated using equation (3) and found 0.37 .

$$
A=\tau_{T}\left(1-\rho_{B}\right)
$$

where, $\tau_{T}$ is the fraction of laser energy transmitted through the top material (PVDF), which is taken as 0.85 . And $\rho_{B}$ is the fraction of laser energy reflected from the top surface of the bottom material (Ti). The refractive index (real part of complex index of refraction) of the $\mathrm{Ti}$ at $804.9 \mathrm{~nm}$ wavelength is 2.90 , and thus, the reflection coefficient, $\rho_{B}$, is 0.56 . The reflection from the top surface of the PVDF film is negligible and, therefore, not considered in calculating parameter $A$.

\subsection{Initial condition and boundary condition}

At the initial condition when time $=0 \mathrm{sec}$, the workpiece has uniform ambient temperature. The environmental temperature is chosen as $300 \mathrm{~K}\left(27^{\circ} \mathrm{C}\right)$. 
In the laser welding process, the workpiece exchanges heat with the medium around it due to convection and radiation, because there is intense difference in the temperature between its boundary and ambience. The surface, exposed to air, transfers the energy by the convective heat transfer mode. This convective heat loss, $q_{c}$, can be expressed as:

$$
q_{c}=h\left(T_{s}-T_{\infty}\right)
$$

where, $h$ is convection heat transfer coefficient, $T_{s}$ is surface temperature, and $T_{\infty}$ is ambient temperature. All the surfaces of the geometry except the top surface, bottom surface and the symmetry plane are assumed to transfer heat by natural convection to the environment since they are exposed to the surroundings. The right vertical side is the plane of symmetry and hence, considered insulated. The convective heat transfer coefficient is taken as $5 \mathrm{~W} / \mathrm{m}^{2}-\mathrm{K}$ (Mayboudi et al., 2005).

Thermal radiation from the top surface of the Ti foil is also considered as boundary condition. The radiant heat loss, $q_{r}$, can be expressed as:

$$
q_{r}=\sigma \varepsilon\left(T_{s}^{4}-T_{\infty}^{A}\right)
$$

where, $\sigma\left(5.67 \times 10^{-8} \mathrm{~W} / \mathrm{m}^{2}-\mathrm{K}^{4}\right)$ is Stefan Boltzmann constant, and $\varepsilon$ is the emissivity. Emissivity of a material is the relative ability of its surface to emit energy by radiation. It is the ratio of the radiation emitted by a surface to the radiation emitted by a blackbody at the same temperature. The emissivity of pure titanium is 0.63 , which is obtained from www.matweb.com.

\subsection{Loading and solution}

A distributed heat flux (Gaussian) is applied on the material body on which the laser is being focused. The heat flux is calculated as a function of beam dimensions, laser power and the fraction of laser energy absorbed by the absorbent part, using Equation (2). A look-up table is made to create the heat flux input file in the form of a 2-D array and is a function of $\mathrm{x}$ - and y-coordinates. The moving load is implemented by a subroutine implemented in APDL language. The load position and magnitude are computed at the beginning of each cycle in the loop. When the load moves to the next load step, the former load step is deleted. In order to ensure convergence, the heat loads are imposed by small increments using *DO loop command. The process continues until the beam reaches the end of the specimen. The resulting effect mimics a continuously moving laser beam with a specified speed and heat flux value. The time step required for a specific laser beam speed can be determined by dividing the distance between the two consecutive elements sets with the beam speed. The load moves with time. Thus, a nodal temperature history is obtained as a function of time. The time is calculated based on the welding speed and heat flux location. The transient heat transfer model is then run and nodal temperature is documented as a function of time for use in the subsequent steps.

\section{Result and Analysis}

The distribution of the temperature field in laser transmission welding of PVDF to Ti is simulated by the commercial multiphysics FEA software, ANSYS $^{\circledR}$ in this paper. Running the developed model with described input parameters with a $2.66 \mathrm{GHz}$ Core $^{\mathrm{TM}} 2$ Duo processor and 3.0 GB of RAM takes approximately 254 minutes of solution time.

\subsection{The thermal simulation of laser welding temperature field}

On the condition of moving heat flux, the temperature distribution of the workpiece changes quickly with time and space. Figure 4 is the plot of isothermal contours at the symmetry plane at $0.2625 \mathrm{~s}$. The highest temperature of $551.96 \mathrm{~K}$ is predicted at the symmetry plane, at the weld interface. Taking into consideration the melting temperature of PVDF, $443 \mathrm{~K}$, the region in the contours plot in red color shows the molten zone of the PVDF foil. Whereas, the maximum temperature achieved during welding is far below the melting temperature of titanium, thus, no melting occurs in the Ti foil.

Figure 5 shows the temperature distribution along the laser irradiation path at the weld interface at $0.2625 \mathrm{~s}$, when the position of the laser beam center is $x=4 \mathrm{~mm}, y=3.5 \mathrm{~mm}$. It can be shown from figure 5 that the thermal field changes as the laser source moves along, and the melt pool moves along with the laser source. The maximum temperature is attained at the laser beam center. It is further observed that the temperature gradient in the leading side of the advancing laser source is much steeper than that in the trailing side.

Figure 6 shows the maximum temperatures achieved at different beam position along the travelling path as a function of time. It can be observed that the maximum temperature increased over a time and then goes to balance, namely it goes into quasi-steady state. Figure 7 confirms that the distribution of temperature field becomes quasi-steady at $0.1688 \mathrm{~s}$. 


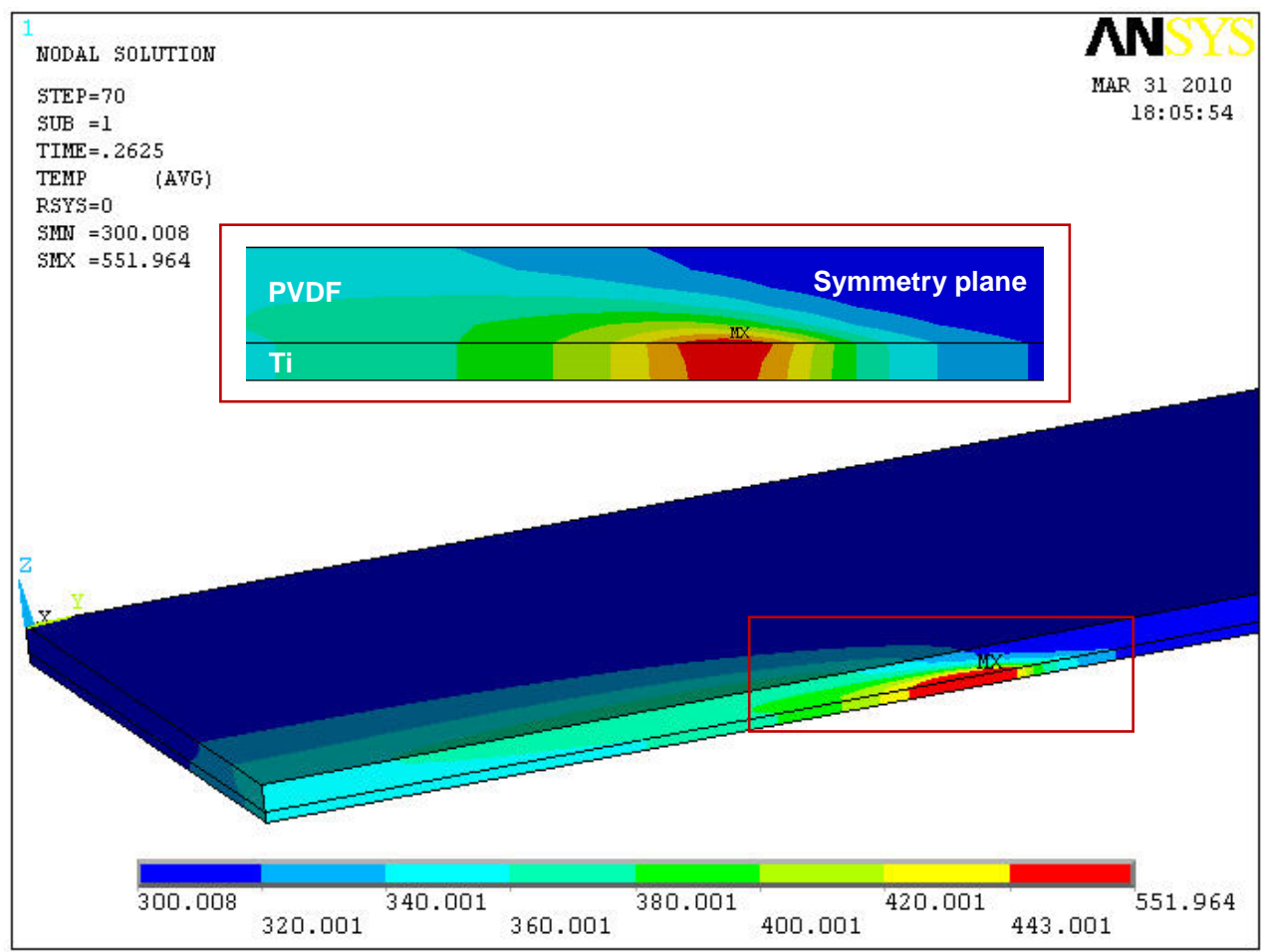

Figure 4. Temperature contours at the symmetry plane (Y-Z plane) at $0.2625 \mathrm{~s}$

\section{P0ST1}

STE $P=70$

SUB $=1$

TIME $=.2625$

PATH PLOT

NOD $1=128586$

NOD $2=136652$

YDIRECT

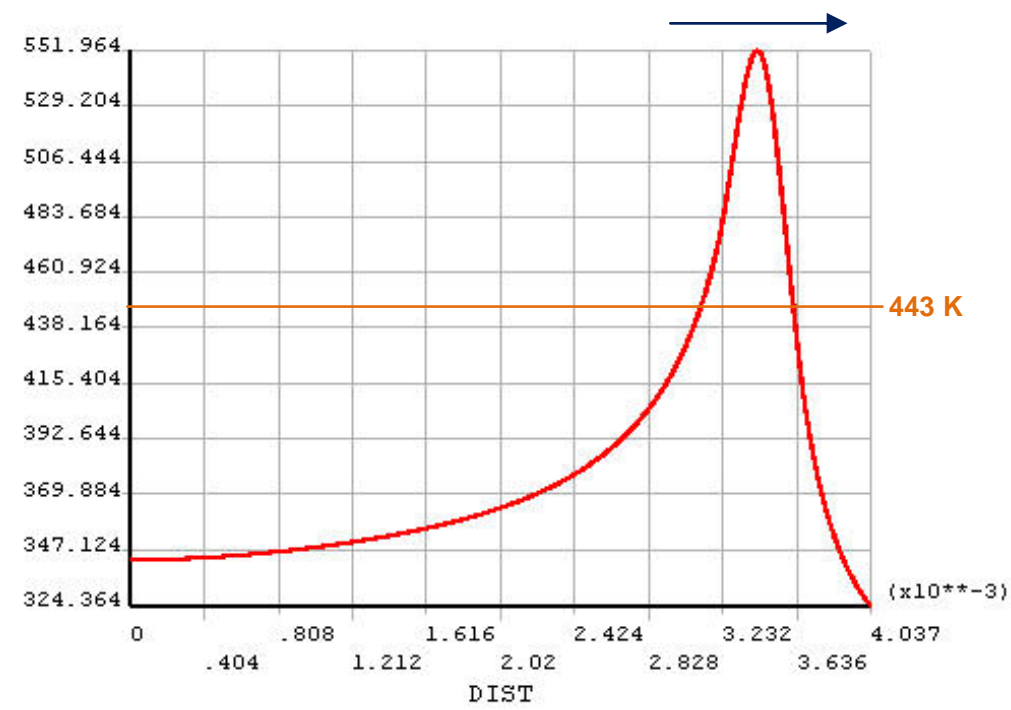

Figure 5. Temperature distribution on the interface along the irradiation path at $0.2625 \mathrm{~s}$ (beam center at $\mathrm{x}=4.0 \mathrm{~mm}, \mathrm{y}=3.5 \mathrm{~mm}$ ) 


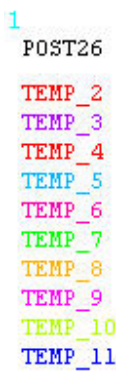

NN

APR 32010

$19: 25: 47$

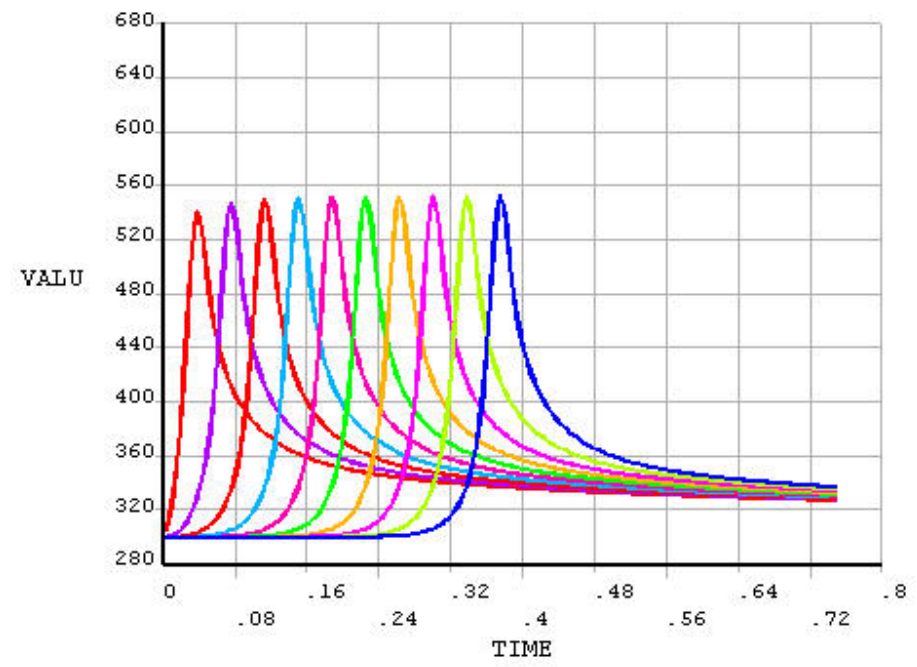

Figure 6. Time vs. Temperature plot for different load steps

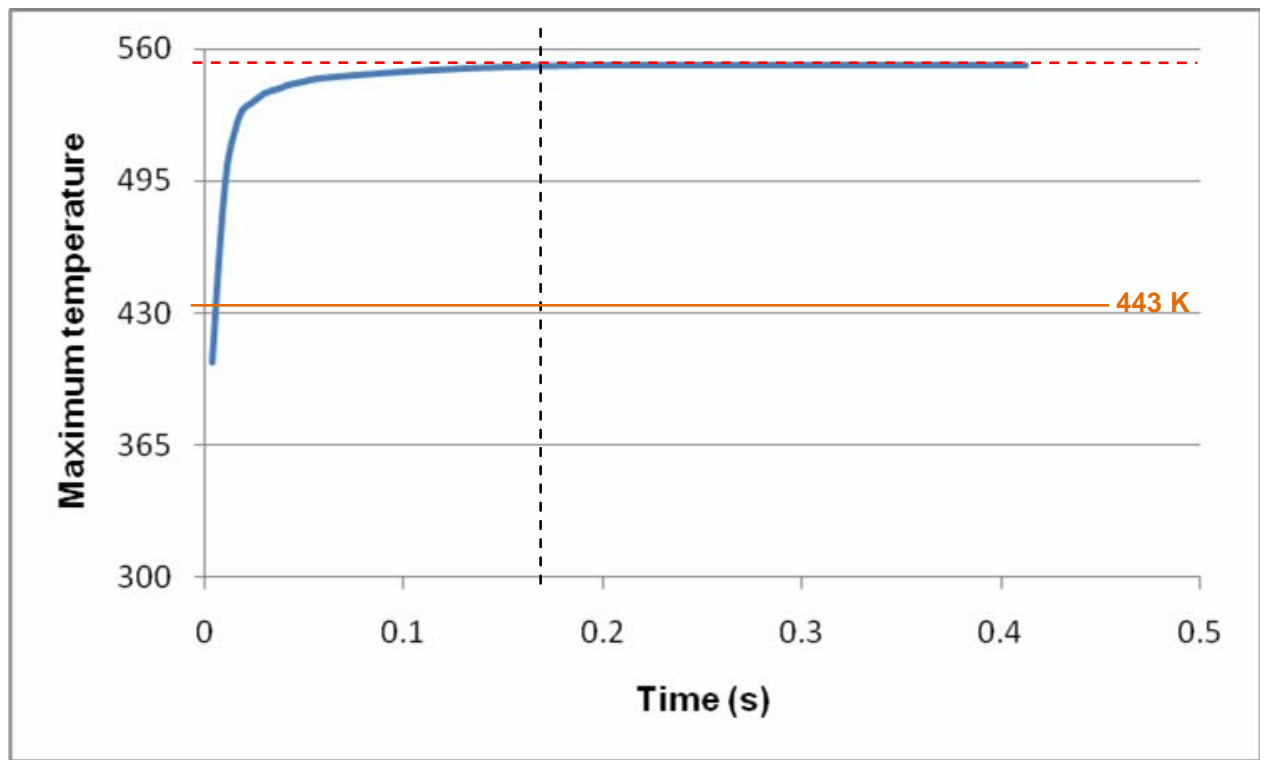

Figure 7. Plot of maximum temperature for over the period of time

It can be postulated that the weld dimensions will be consistent, once the temperature distribution of weld zone reaches steadystate at $0.1688 \mathrm{~s}$. Hence, the weld dimensions are calculated at $0.2625 \mathrm{~s}$.

\subsection{Thermal simulation of the weld pool}

Figure 8 shows the melt pool of PVDF foil in red color, at the symmetry plane. The predicted temperature at weld interface is above the melting temperature of PVDF and, as a result, bonding occurs between PVDF and Ti foils. In the welding process, the molten area of PVDF reacts chemically with the titanium surface at the Ti / PVDF interface and thus forms the bonding zone. Good quality welding is possible only when the temperature at the joint interface is above the melting temperature (443 K) but below the decomposition temperature $(648 \mathrm{~K})$ of the PVDF. 
Figure 9 shows the temperature distribution along the $\mathrm{Z}$ axis at the center of the laser beam, at $x=4.0 \mathrm{~mm}$ and $y=3.5 \mathrm{~mm}$. The molten depth in the PVDF foil can be calculated with the data shown in the picture. Using this distance vs. temperature plot the molten depth of $24 \mu \mathrm{m}$ is obtained.

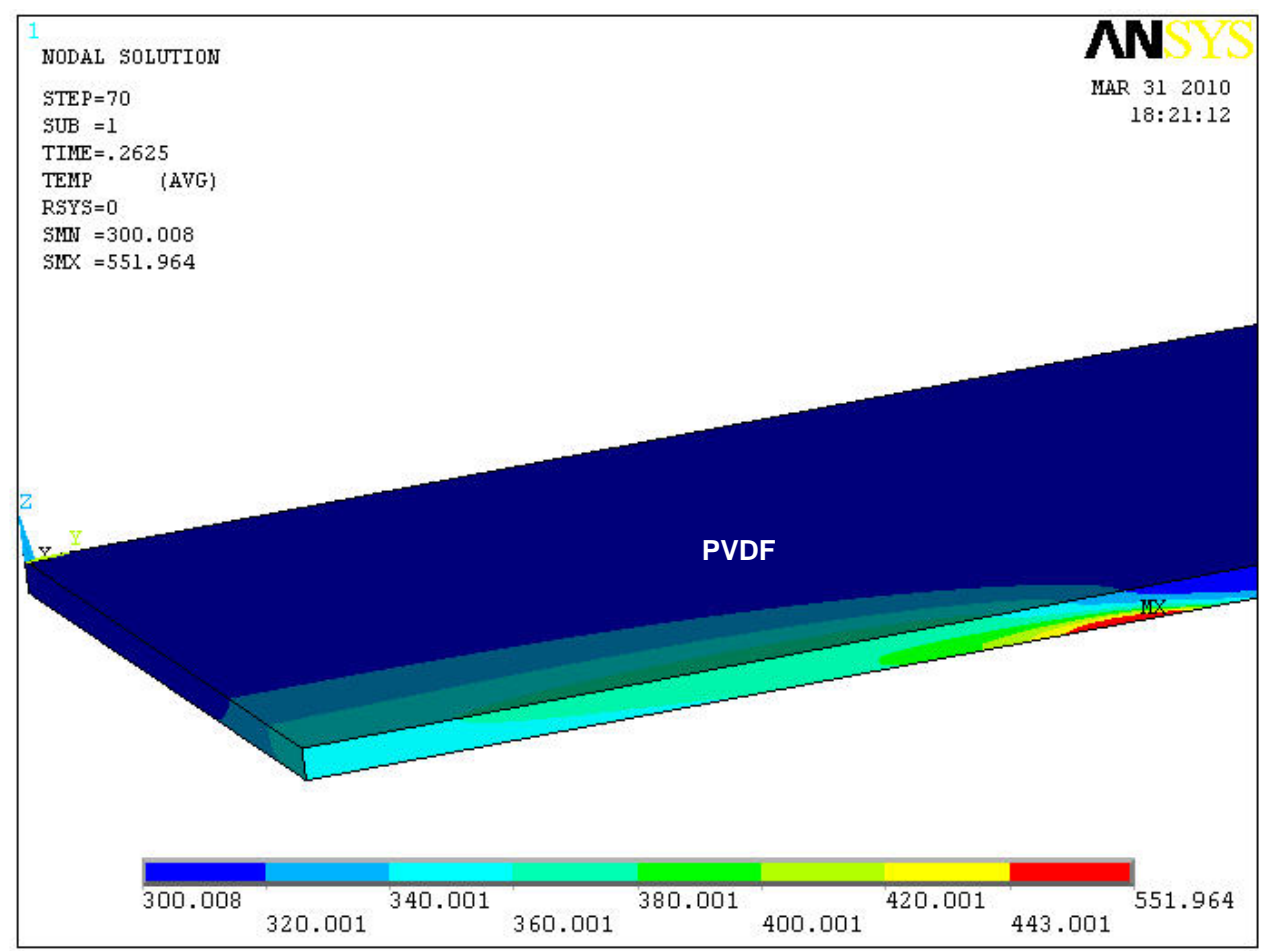

Figure 8. Temperature contours at the symmetry plane (Y-Z plane) at $0.2625 \mathrm{~s}$ (material: PVDF)

POST1

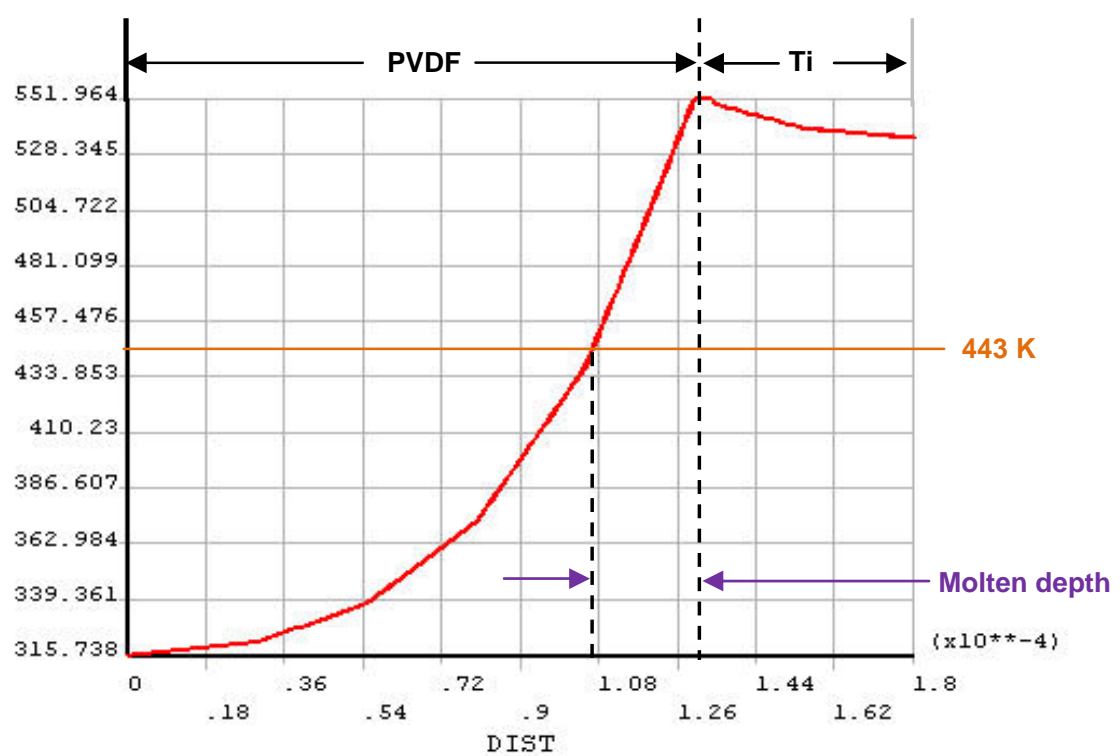

Figure 9. Temperature distribution along the $Z$ axis at $0.2625 \mathrm{~s}$ (beam center at $x=4.0 \mathrm{~mm}, y=3.5 \mathrm{~mm}$ ) 
The temperature distribution at the weld interface at $0.2625 \mathrm{~s}$ is shown in figure 10 . It can be observed that the isotherm patterns appear as near elliptic. The temperature zone presented in red color at the weld interface (X-Y plane) shows the molten area of PVDF surface.

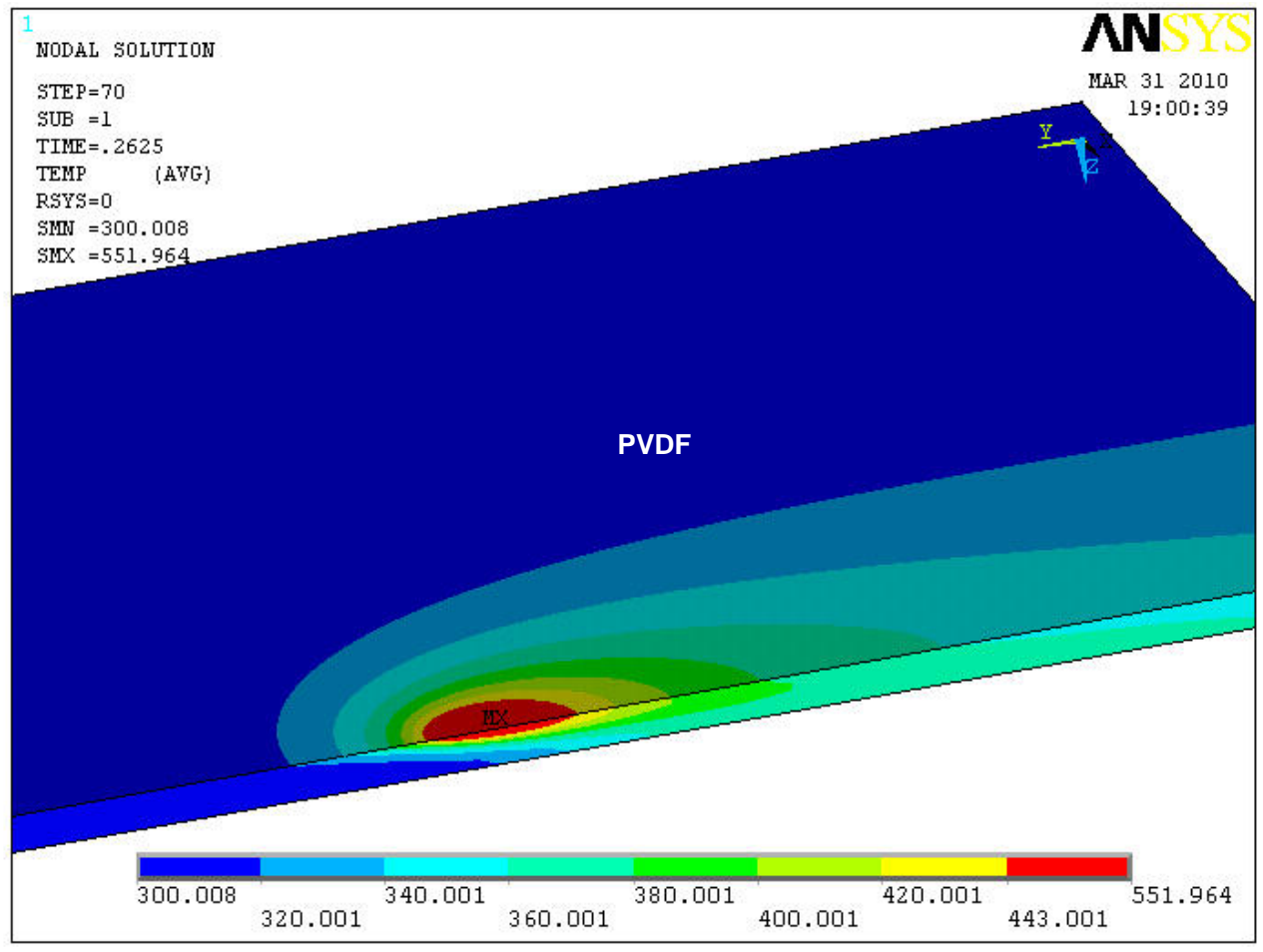

Figure 10. Temperature contours at the weld interface (X-Y plane) at $0.2625 \mathrm{~s}$ (material: PVDF, bottom side up)

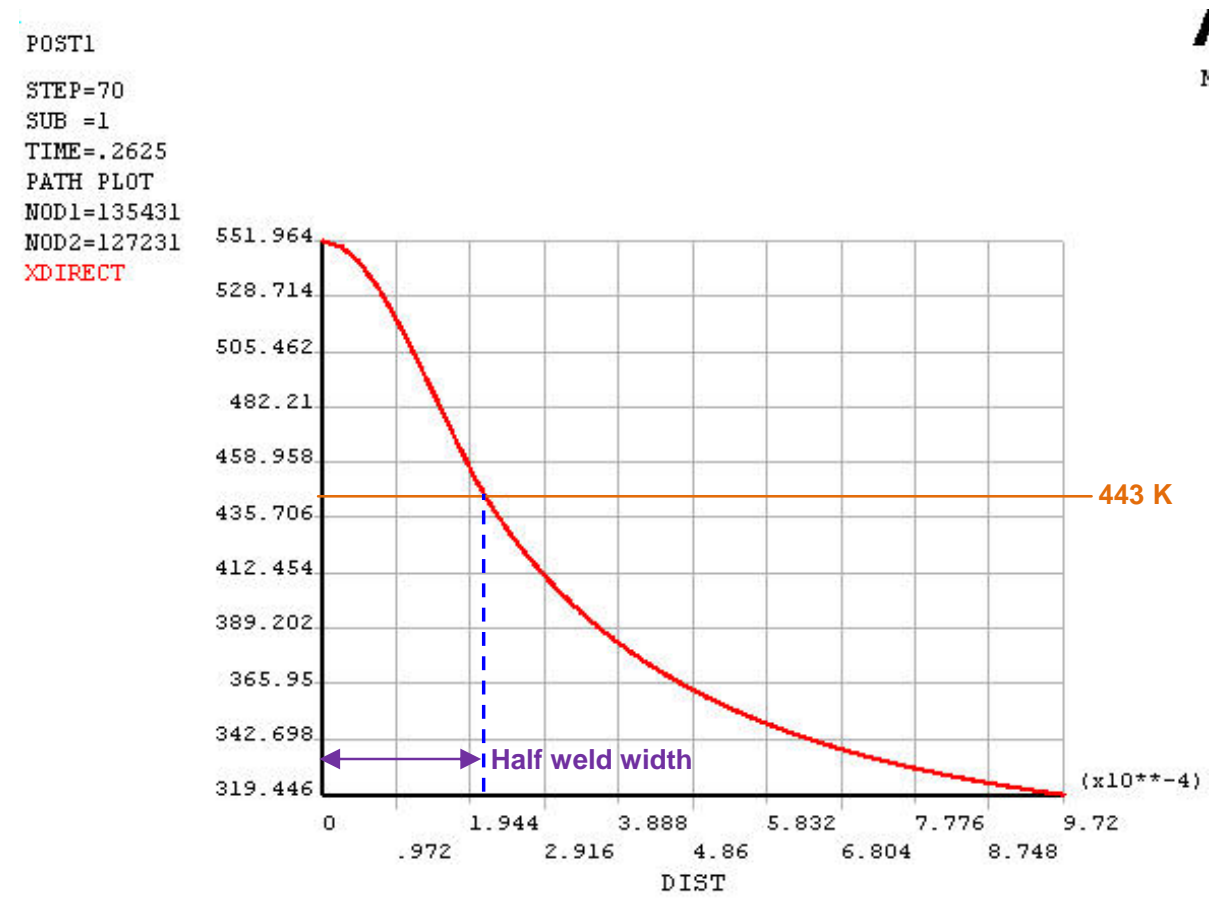

Figure 11. Temperature distribution along the $\mathrm{X}$ axis at $0.2625 \mathrm{~s}$ (beam center at $x=4.0 \mathrm{~mm}, y=3.5 \mathrm{~mm}$ ) 
Figure 11 shows the variation of the temperature along a line transverse to the scanning path, with its distance from the center of the weld pool (beam center at $x=4.0 \mathrm{~mm}, y=3.5 \mathrm{~mm}$ ). It can be seen from the figure that the temperature drops rapidly with distance from the center of the laser beam along $x$. It is observed that the temperature falls by $185 \mathrm{~K}$ at a node $0.5 \mathrm{~mm}$ away from the center. The weld half width can be calculated with the data shown in this picture. Considering the melting temperature of PVDF to be $443 \mathrm{~K}$, the weld half width of $216 \mu \mathrm{m}$ is obtained from the figure 11 .

\section{Conclusions}

A model involving moving distributed heat flux for laser transmission metal to plastic welding has been implemented into FE thermal simulations to predict temperature field during the process. Further, experiment is required so as to corroborate the proposed heat flux model and to establish quantitative correlations between FE simulations and experiments.

This numerical model uses an energy balance method to calculate the temperature at nodes throughout the materials at specified time steps. The developed 3-D model is capable of predicting the transient temperature field in the specimen and the weld dimensions. In addition, this model can also be used to determine a suitable combination of laser power and laser welding speed for welding a specific set of dissimilar material. Furthermore, this thermal model's flexibility to take on a variety of materials, boundary conditions, optical properties, and geometry makes it suitable for its application in parametric studies of a wide range of laser transmission metal to plastic welding problems.

A more general 3-D finite element model for laser transmission metal to plastic welding process is underway including the effect of contact resistance due to the presence of any air gap at the weld interface.

\section{Nomenclature}

FEA Finite element analysis

LTW Laser transmission welding

PVDF Polyvinylidene fluoride

$\mathrm{Ti}$ Titanium

APDL $\quad$ ANSYS $^{\circledR}$ Parametric Design Language

$\mathrm{Cr} \quad$ Chromium

Ta Tantalum

Al Aluminium

MEMS Micro-electro-mechanical system

$\lambda \quad$ Wavelength of laser radiation

$k_{x}, k_{y}, k_{z}$ Thermal conductivity in the $x, y$ and $z$ directions

$C \quad$ Specific heat capacity

$\rho \quad$ Density

$t \quad$ Time

$q(x, y, z, t)$ Internal heat generation rate per unit volume

$t_{m} \quad$ Melting point

$A \quad$ Fraction of laser energy absorbed in the absorbing material

$P \quad$ Laser beam power

$r_{0} \quad$ Laser beam radius

$r \quad$ Radial distance of any point from beam center

$x_{s}, y_{s} \quad$ Cartesian coordinates of a point on the surface of the material

$\tau_{T} \quad$ Fraction of laser energy transmitted through the material

$\rho_{B} \quad$ Fraction of laser energy reflected from the material

$q_{c} \quad$ Convective heat loss

$h \quad$ Convection heat transfer coefficient

$T_{s} \quad$ Surface temperature of the material

$T_{\infty} \quad$ Ambient temperature

$q_{r} \quad$ Radiant heat loss

$\sigma \quad$ Stefan Boltzmann constant $\left(5.67 \times 10^{-8} \mathrm{~W} / \mathrm{m}^{2}-\mathrm{K}^{4}\right)$

$\varepsilon \quad$ Emissivity

\section{References}

Acherjee, B., Misra, D., Bose, D. and Venkadeshwaran, K., 2009. Prediction of weld strength and seam width for laser transmission welding of thermoplastic using response surface methodology. Optics \& Laser Technology, Vol. 41, No. 8, pp. 956967. 
ANSYS $^{\circledR} 10$ Manual.

Becker, F. And Potente, H., 2002. A step towards understanding the heating phase of laser transmission welding in polymers.

Polymer Engineering and Science, Vol.42, No. 2, pp. 365-374.

Coelho, J.M.P., Abreu, M.A. and Rodrigues, F.C., 2008. Modeling the spot shape influence on high-speed transmission lap welding of thermoplastic films. Optics and Lasers in Engineering, Vol.46, pp.55-61.

Dutta Majumdar, J. and Manna, I., 2003. Laser processing of materials. Sadhana - Academy Proceedings in Engineering Sciences, Vol. 28, pp. 495-562.

Ilie, M., Kneip, J.C., Mattei, S. and Nichici, A., 2005. Effects of laser beam scattering on through-transmission welding of Polymers. Proceedings of the 24th International Congress on Applications of Lasers \& Electro-Optics, Miami, Florida, USA, Vol.98, pp. 388-393.

Incropera, F.P. and DeWitt, D.P., 1996. Introduction to Heat Transfer. $3^{\text {rd }}$ ed. John Wiley \& Sons.

Kennish, Y.C., Shercliff, H.R. and McGrath, G.C., 2002. Heat flow model for laser welding of polymers. Proceedings of Annual Technical Conference - ANTEC 2002, San Fransisco, CA, USA, pp. 1132-1137.

Mayboudi, L.S., Birk, A.M., Zak, G. and Bates, P.J., 2005. A 2-D Thermal model for laser transmission welding of Thermoplastics. Proceedings of the 24th International Congress on Applications of Lasers \& Electro-Optics, Miami, Florida, USA, Vol.98, pp. 402-409.

Mayboudi, L.S., Birk, A.M., Zak, G. and Bates, P.J., 2007. Laser transmission welding of a lap-joint: Thermal imaging observations and Three-dimensional finite element modeling. ASME - Journal of Heat Transfer, Vol. 129, pp. 1177-1186.

Mayboudi, L.S., Birk, A.M., Zak, G., and Bates, P.J., 2007. A 3-D Thermal Model of Laser Transmission Contour Welding for a Lap Joint. Proceedings of Annual Technical Conference - ANTEC 2007, Cincinnati, OH, USA, pp. 2831-2817.

Sultana, T., Georgiev, G.L., Auner, G., Newaz, G., Herfurth, H.J. and Patwa, R., 2008. XPS analysis of laser transmission microjoint between poly (vinylidene fluoride) and titanium. Applied Surface Science, Vol. 255, pp. 2569-2573.

Sultana, T., Georgiev, G.L., Baird, R.J., Auner, G.W., Newaz, G., Patwa, R. and Herfurt, H.J., 2009. Study of two different thin film coating methods in transmission laser micro-joining of thin Ti-film coated glass and polyimide for biomedical applications. Journal of the Mechanical Behaviour of Bio Medical Materials, Vol. 2, pp. 237-242.

Taha, Z.A., Roy, G.G., Hajim, K.I. and Manna, I., 2009. Mathematical modeling of laser-assisted transmission lap welding of Polymers. Scripta Materialia, Vol. 60, pp. 663-666.

Van de Ven, J.D. and Erdman, A.G., 2007. Laser transmission welding of thermoplastics-Part I: Temperature and Pressure Modeling. Journal of Manufacturing Science and Engineering, Vol. 129, pp. 849-858.

\section{Biographical notes}

Bappa Acherjee is presently a Research Scholar in the Department of Production Engineering at Jadavpur University, Kolkata, India. He completed his graduation in Production Engineering from West Bengal University of Technology, Kolkata in 2006 and post graduation in Manufacturing Technology from National Institute of Technical Teachers' Training and Research, Kolkata, India in 2009. His research interest includes laser material processing and finite element method in manufacturing processes. He is a Member of ASME.

Arunanshu S. Kuar is the Reader in Production Engineering Department, Jadavpur University, Kolkata, India. He has been awarded Ph.D. in the area of laser beam micro-machining. He has published more than 40 National and International journals and conference papers and guided a number of Post Graduate and Doctoral Research Work.

Souren Mitra is the Head of Production Engineering Department of Jadavpur University, Kolkata, India. His research areas include nontraditional machining, micro-machining and advanced manufacturing systems. He has published more than 60 research papers in international and national journals and conferences. Several $\mathrm{PhD}$ and $\mathrm{M}$. ProdE theses have been completed under his guidance. He is a recipient of the Research Award of UGC, Government of India, and has successfully completed four sponsored research projects as the principal investigator. He has attended several international conferences abroad.

Dipten Misra is the Director of School of Laser Science and Engineering, Jadavpur University, Kolkata, India. He has 25 years of teaching experience. He has published a number of research papers in national and international journals. His research interests are heat and mass transfer, computational fluid dynamics and laser material processing.

Received January 2010

Accepted March 2010

Final acceptance in revised form May 2010 